\title{
Ueber die embryonale
} Hand- und Fuss-Skelett-Änlage bei den Crocodiliern, sowie über ihre Beziehungen zur Vogel-Flügelanlage und zur ursprünglichen Tetrapoden-Extremität

\author{
von

\section{Hans STEINER,} \\ Zürich.
}

(Aus dem zoolo gisch-vergl. anatomischen Institut der Universität Zürich.)

Mit 10 Textfiguren.

Seit den vorzüglichen Untersuchungen Fr. v. HuEnE's über die Archosaurier, 1914, ist es immer wahrscheinlicher geworden, dass die grossen Reptilienordnungen der Thecodontia, der Ornithischia und Saurischia, der Pterosauria und der Crocodilia zusammen mit den Vögeln zu einem engeren Formenkreise gehören, welcher einen gemeinsamen Ursprung besessen haben muss. Diese verwandtschaftlichen Beziehungen prägen sich deutlich in vielen Merkmalen der Organisation dieser an und für sich recht divergenten Tiergruppen aus, unter anderem auch im Bau der Extremitäten. Recht auffällig ist in diesem vor allem die schon frühzeitig nachweisbare Tendenz, die nach vorn gerichteten, radialen oder tibialen Fingerstrahlen kräftiger und stärker auszubilden als die nach hinten gerichteten, ulnaren Finger oder fibularen Zehen. In einer Studie über die ontogenetische und phylogenetische Entwicklung des Vogelflügelskelettes habe ich 1922 bereits eingehender auf diese Verhältnisse hingewiesen. Damals ist auch die Extremität der Crocodilier, als der einzigen heute noch lebenden, nächsten Verwandten der Vögel, weitgehend zum Vergleiche herbeigezogen worden, 
wobei sich dieser Vergleich allerdings nur auf das Skelett des fertigen Individuums beschränken musste, weil eine einlässliche ontogenetische Untersuchung seiner Entwicklung nicht vorlag. Durch die Vermittlung meines leider inzwischen verstorbenen Freundes Prof. Dr. E. Huber und von Herrn Prof. Dr. A. Schultz, beide an der Johns Hopkins Medical School Baltimore, habe ich seitdem von Herrn Prof. Dr. G. L. Streeter, Direktor des Department of Embryology, Carnegie Institution of Washington in Baltimore, eine vollständige Embryonen-Reihe von Caimanen in freundlichster Weise zur Untersuchung der Ontogenese des Handund Fuss-Skelettes der Crocodilier zur Verfügung erhalten. Die Untersuchung ergab teilweise ganz andere Zusammenhänge, als wie ich sie 1922 vermutet hatte. Diese zeigen aber noch viel deutlicher die engen Beziehungen, welche zwischen den heute noch lebenden Archosauriern, den Vögeln und den Crocodiliern, bestehen und gestatten zudem, den Anschluss an die Verhältnisse bei der primitivsten bisher bekannt gewordenen Tetrapoden-Extremität zu gewinnen. Im Nachfolgenden soll auf die Hauptresultate dieser embryologischen Untersuchung hingewiesen werden; die ausführliche Publikation wird andernorts erfolgen.

Die allererste Beschreibung des Hand- und Fuss-Skelettes der Crocodilier geht, wie die meisten Angaben über das Skelett der Wirbeltiere, auf Cuvier, 1824, und Meckel, 1824, zurück. Die ersten vergleichend-anatomischen Angaben lieferte jedoch Gegenbaur, 1864. Diese haben sich fast unverändert bis auf den heutigen Tag in sämtlichen Lehrbüchern erhalten. Nach GEgenBAUR findet man im Carpus der fünfzehigen Saurier im allgemeinen 8 distinkte Elemente, nämlich in der proximalen Reihe: ein Radiale, ein Centrale und ein Ulnare; in der distalen Reihe: 5 Carpalia. Das für das Basipodium der Urodelen so charakteristische Intermedium soll fehlen und konnte von GEGEnBAUR auch bei Embryonen von Lacerta nicht gefunden werden; wohl aber wies Born, 1876, ein solches bei Lacerta nach. Im übrigen sollten sich Schildkröten, Eidechsen und Krokodile nach GEgenbaur im Baue ihres Carpus und Tarsus ziemlich unvermittelt gegenüberstehen, da Uebergangsformen fehlten. Im Crocodilier-Carpus beschreibt er 6 verschiedene Stücke (vergl. Figur 1): in der proximalen Reihe ein überaus merkwürdig gestaltetes, längliches Radiale (r), ferner ein Ulnare $(u)$ und ein accessorisches Sesam- 
bein (s); ein Intermedium fehlt wiederum; in der distalen Reihe: zunächst ein an das Radiale anstossendes, knorpeliges Centrale (c), darunter versteckt (was Cuvier und MEckel übersahen) ein verschmolzenes Carpale $1+2$, und endlich ein verschmolzenes Carpale $3+4+5$. Spätere Untersucher, wie Hoffmann, 1890, stimmen im wesentlichen mit Gegenbaur überein. Hoffuann untersuchte auch jüngere Exemplare von Gavialis, Alligator und Crocodilus und stellte im Radiale und Ulnare nur je einen Knochenkern fest; vom Intermedium fehlte also wiederum jede Spur. Ferner fand er nur einen Knochenkern im Carpale $3+4+5$. Hoffmann macht speziell auf die Tatsache aufmerksam, dass das Metacarpale I unmittelbar dem Centrale anliegt und mit seiner
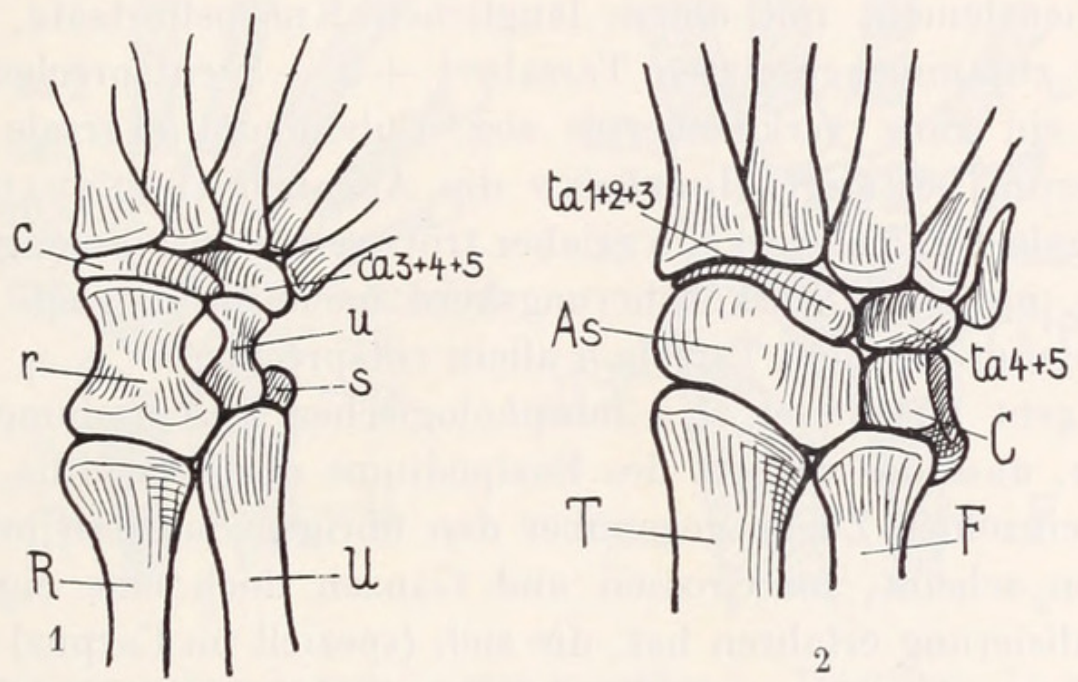

2

Fig. 1. - Carpus eines Alligator mississipiensis; nach Gegenbaur, 1864.

(Erklärung der in den Textfiguren gebrauchten Abkürzungen siehe S. 395).

Fig. 2. - Tarsus eines Alligator mississipiensis; nach Gegenbaur, 1864.

Basis aus der Reihe der übrigen Metacarpalia herausspringt; es sollte deshalb das an dieser Stelle liegende Carpale 1 mit dem Metacarpale I verwachsen sein, sodass also das Gegenbaur'sche Carpale $1+2$ nur aus dem Carpale 2 bestehen würde.

Im Tarsus tritt nach Gegenbaur bei allen Sauriern in der proximalen Reihe nur ein einziges Knochenstück auf, das allerdings seine zusammengesetzte Natur durch eine breitere tibiale Hälfte und eine kleinere fibulare Hälfte zu erkennen gibt, wie denn schon Cuvier bei Monitor feststellte, dass es aus zwei Stücken bestehe. Der tibiale Teil sollte dem sog. Astragalus entsprechen, also einem verschmolzenen Tibiale + Intermedium, die fibulare Partie einem 
Calcaneus (Fibulare). In der distalen Reihe sind nur zwei Tarsalia erhalten, ein Tarsale 3 und ein verschmolzenes Tarsale $4+5$. Da jedoch die Metatarsalia I und II direkt an den sog. Astragalus anstossen, sollen in ihren basalen Endstücken die Tarsalia 1 und 2 enthalten sein. Im Tarsus der Crocodilier (vergl. Fig. 2) stellte Gegenbaur in der proximalen Reihe, abweichend von den übrigen Sauriern, zunächst zwei distinkte Stücke fest, was nur als ein primitives Merkmal bewertet werden konnte. Beim grösseren Element musste es sich um ein Astragalo-Naviculare (Tibiale + Intermedium + Centrale) handeln, beim kleineren um einen separaten Calcaneus (Fibulare). (As und Cin Fig. 2.) In der distalen Reihe beschreibt Gegenbaur ein dem Astragalus anliegendes Knochenelement mit einem länglichen Knorpelfortsatz, welches einem zusammengesetzten Tarsale $1+2+3$ entsprechen sollte, sowie ein ganz verknöchertes sog. Cuboideum (Tarsale $4+5$ ). Wiederum bestätigt Hoffmann die Angaben Gegenbaur's; im Astragalo-Naviculare kann er aber trotz seiner zusammengesetzten Natur nur einen Verknöcherungskern nachweisen, auch soll das Cuboideum nur dem Tarsale 4 allein entsprechen.

Es geht aus dieser rein morphologischen Beschreibung bereits hervor, dass das Skelett des Basipodiums der Crocodilia, obwohl es in einzelnen Zügen gegenüber den übrigen Sauriern primitiver zu sein scheint, im Grossen und Ganzen doch eine eigenartige Spezialisierung erfahren hat, die sich (speziell im Carpus) in einer besonderen Gestaltung und teilweisen Reduktion seiner Elemente kundgibt. Eine genaue ontogonetische Untersuchung versprach deshalb von vornherein interessante Einzelheiten aufzudecken, ergab doch schon die Prüfung jugendlicher Individuen, dass bei ihnen gegenüber dem verknöcherten, adulten Basipodium ursprünglichere Verhältnisse angedeutet sind (vergl. Fig. 3 und 4). Im jugendlichen Carpus (Fig. 3) zeigt das Gegenbaur'sche Radiale (crp) an der Aussenseite seiner Artikulation mit dem Radius ein kleines, rudimentäres Knorpelelement (r) und gegen die Ulna hin einen breiten Knorpelfortsatz (i). In jedem der drei proximalen Elemente tritt ein selbständiger Knochenkern auf. Die Gestaltung des sog. Centrale weist deutlich durch zwei merkliche Anschwellungen auf seine zusammengesetzte Natur hin. Das gleiche gilt für das Carpale $3+4+5$, bei welchem namentlich für den fünften Fingerstrahl ein deutlicher Fortsatz ausgebildet ist. Carpale 2 liegt 
als ein selbständiges Element vor. Sehr auffällig ist das Vorspringen der Basis des Metacarpale I, in welcher tatsächlich mit Hoffmann das Carpale $1 \mathrm{zu}$ vermuten ist. Im jugendlichen Tarsus (Fig. 4) zeigt zunächst das sog. Astragalo-Naviculare eine typische Konfiguration, welche wiederum durch einen breiten Fortsatz mit Ansatzfläche an der Fibula auffällt. Ausser einem grossen Ossifikationszentrum konnte in einem Falle (dargestellt in Fig. 4) ein weiterer kleiner Knochenkẹn distal von jenem nachgewiesen werden. Der sog. Calcaneus (Fibulare) ist deutlich als selbständiges Element zu erkennen; ausserdem erscheint jedoch in seinem

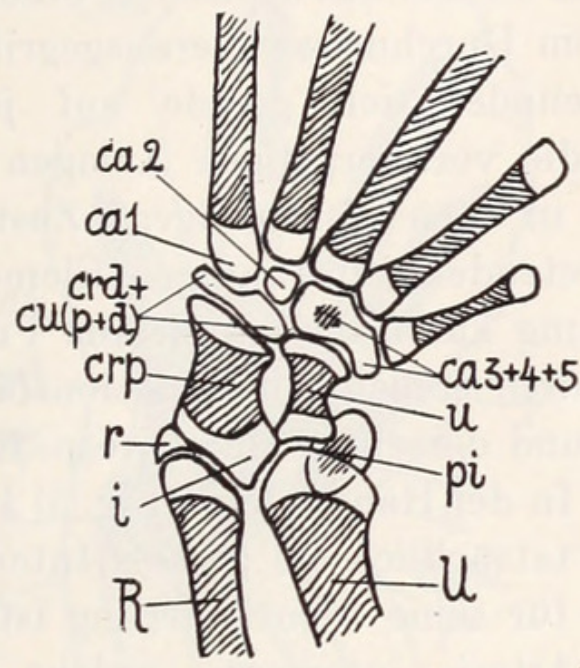

3

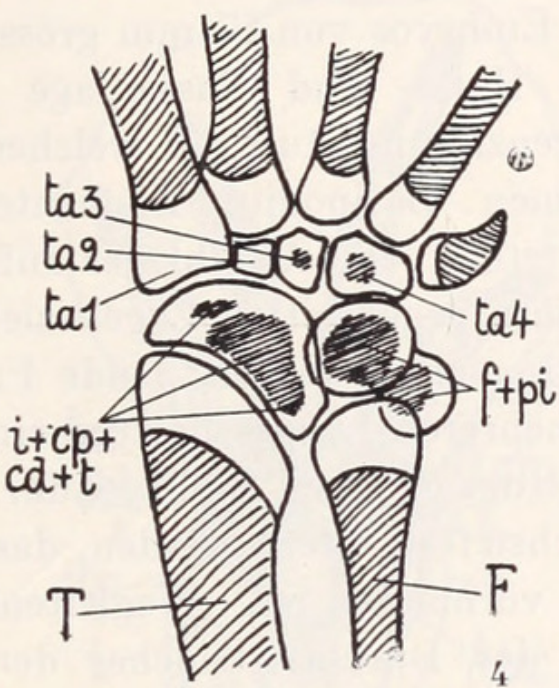

4

FIG. 3 - Carpus eines jungen Caiman crocodilus L. Totallänge $23 \mathrm{~cm}$. Original. Verknöcherungszentren schraffiert angegeben.

FIG. 4. - Tarsus eines jungen Caiman crocodilus L. Totallänge $23 \mathrm{~cm}$. Original.

plantarwärts gerichteten, sog. Fersenhöcker ein weiteres selbständiges Ossifikationszentrum nebem dem Hauptkern, welches wohl das Pisiforme darstellt, während der Hauptkern dem eigentlichen Fibulare entspricht. In der distalen Reihe sind deutlich drei selbständige Tarsalia, nämlich 2, 3 und $4+5$, nachweisbar. Auch hier im Fusse springt die Basis des Metatarsale I vor, sodass in ihr wiederum das Tarsale $1 \mathrm{zu}$ vermuten ist.

Zur Ontogonese der Crocodilier-Extremität hat im wesentlichen bisher blos Kuekenthal, 1893, einen Beitrag geliefert. Ihm gelang der Nachweis, dass an Stelle des späteren einheitlichen, als Centrale gedeuteten Elementes in der embryonalen Hand des Krokodils drei noch deutlich voneinander zu unterscheidende Elemente vorhanden 
sind; ferner, dass das Carpale $3+4+5$ tatsächlich durch die Verschmelzung der selbständig angelegten Carpalia 3, 4 und 5 entsteht. Im sog. Radiale findet er einen vom Hauptteile getrennten, zwischen Ulna und Radius gelegenen Fortsatz, in welchem er das Intermedium vermutet.

Für die eigenen Untersuchungen standen mir 18 Embryonen aufeinanderfolgender Entwicklungsstufen zur Verfügung, sowie mehrere jugendliche Exemplare, welche mindestens zwei verschiedenen Arten, aber alle zur Gruppe der sog. Caimane gehörten (das Material wurde auf Veranlassung von Dr. A. M. ReEse in British Guiana gesammelt). Aus dieser Serie sei hier das typischste Stadium eines Embryos von 17 mm grösstem Durchmesser herausgegriffen. Seine Hand- und Fussanlage befindet sich gerade auf jener Differenzierungsstufe, in welcher die vorknorpeligen Anlagen der einzelnen Basipodium-Elemente in den knorpeligen Zustand übertreten. Die Anzahl der auftretenden selbständigen Elemente und ihre gegenseitige Lagebeziehung kann am besten aus Fig. 5 und 6 ersehen werden; beide Figuren stellen Kombinationsbilder aus mehreren Längsschnitten ein und derselben Hand- resp. Fussanlage des gleichen Embryos dar. In der Handanlage (Fig. 5) kann zunächst festgestellt werden, dass tatsächlich ein grosses Intermedium vorhanden ist. Wegleitend für seine Identifizierung ist die Lage des Durchtrittsloches der Arteria interossea, welche sich schon bei den Urodelen typisch zwischen dem Intermedium und dem Ulnare befindet, ferner die breite Ansatzfläche mit der Ulna. Distal vom Intermedium, im gleichen Gewebekomplex, tritt ein kräftiges Knorpelzentrum auf, das seiner Lage nach nur als ein Centrale proximale, und zwar als Centrale radiale proximale gedeutet werden kann. Ein rudimentärer äusserer Fortsatz des gleichen Gewebekomplexes zum Radius hin enthält einen eigenen Knorpelkern, der als direkte Fortsetzung des Radius nur als das eigentliche Radiale selbst angesprochen werden kann. Es besteht somit das grosse proximale, radiale Carpalelement der Crocodilier zum grössten Teil a us den Anlagendes Intermediums und des Centrale radiale proximale; es stellt also ein verschmolzenes Intermedio-Centrale dar $(\operatorname{crp}+\mathrm{i}+$ Rudiment des eigentlichen Radiale r). Das Ulnare auctorum entspricht auch ontogenetisch dem Ulnare und das accessorische Sesambein 
Gegenbaur's dem typischen Pisiforme der höheren Tetrapoden. In der distalen Reihe kann für jeden Fingerstrahl ein eigenes Carpale nachgewiesen werden; es verschmelzen in der Tat später die Carpalia 3, 4 und 5 miteinander, ebenso das Carpale 1 mit dem Metacarpale I. Im sog. Centrale treten, wie schon KuekenthaL richtig beobachtet hat, drei Elemente auf; von diesen liegt das grösste und am deutlichsten sichtbare direkt dem Ulnare und dem Carpale 4 an. Dieser Lage entsprechend kann es sich nur um ein Centrale ulnare proximale handeln. Für die beiden anderen Ele-
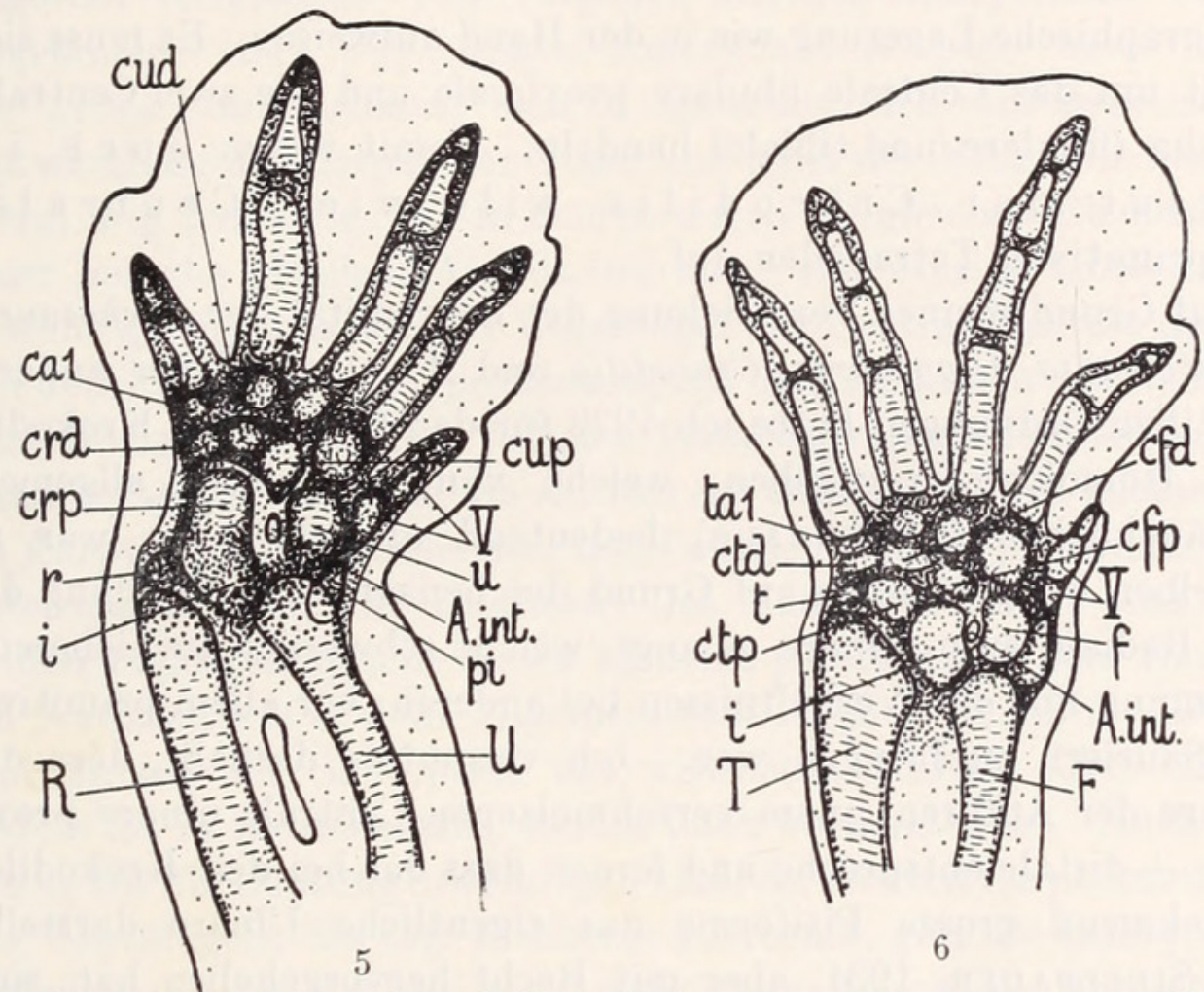

FIg. 5. - Handanlage eines Caiman-Embryos von $17 \mathrm{~mm}$. grösstem Durchmesser. Original. Vorknorpelige selbständige Knorpelzentra hellpunktiert. Fig. 6. - Fussanlage eines Caiman-Embryos von $17 \mathrm{~mm}$.gr. Durchm. Original.

mente folgt damit ungezwungen die Deutung als zwei Centralia distalia und zwar ein radiales und ein ulnares. Damit haben wir in der embryonalen Hand der Crocodilier alle vier Centralia nachgewiesen, welche auch im Carpus der primitivsten Tetrapoden auftreten und zwar, wie gleich noch nachgewiesen werden soll, in einer durchaus ähnlichen Lagebeziehung zueinander.

Was die Fussanlage anbetrifft, so ist an ihr in erster Linie auffällig, in wie weitgehend gleicher, homodynamer Art und Weise das 
Auftreten der einzelnen Tarsalelemente zu jener der Carpuselemente erfolgt (vergl. Fig. 6). Nach dem Fixpunkt der Arteria interossea lässt sich wiederum unzweideutig das grosse Intermedium, ferner das Fibulare und das Pisiforme feststellen. Das Intermedium steht ebenfalls wieder in engerer Beziehung zu einem Centrale tibiale proximale und zu einer rudimentären Anlage des eigentlichen Tibiale. In der distalen Reihe sind fünf selbständige Tarsalia nachweisbar, von welchen das Tarsale 1 wieder mit der Basis des Metatarsale I verschmilzt. Zwischen distaler und proximaler Reihe tauchen wiederum drei Elemente auf, welche die gleiche topographische Lagerung wie in der Hand aufweisen. Es muss sich somit um das Centrale fibulare proximale und die zwei Centralia distalia (fibulare und tibiale) handeln. Somit treten a $\mathrm{u} \mathrm{h} \mathrm{im}$ Tarsus der Crocodilia alle vier Centralia der primitiven Tetrapoden auf.

Auf Grund meiner Vergleichung der Extremität der Archosauria (Thecodontia, Dinosauria, Crocodilia und Aves) mit jener anderer Reptilien-Ordnungen hatte ich 1922 für den Carpus der Krokodile eine Homologie angegeben, welche von der bisher allgemein gebräuchlichen (Gegenbaur) bedeutend abwich. Ich war zu derselben hauptsächlich auf Grund der benachbarten Stellung des sog. Radiale zum Ulnare gelangt, welche schwerlich in Uebereinstimmung mit den Verhältnissen bei anderen, vor allem primitiveren Sauriern zu bringen war. Ich vermutete deshalb, dass das Ulnare der Autoren einem verschmolzenen Centrale ulnare proximale + distale entspreche und ferner, dass das bei den Krokodilen ausnehmend grosse Pisiforme das eigentliche Ulnare darstelle. Wie Sieglbauer, 1931, aber mit Recht hervorgehoben hat, sind bei dieser Homologisierung die topischen Beziehungen zu den Nachbar-Organen (speziell Arteria interossea, Nervus ulnaris, Insertionssehne des Flexor carpi ulnaris) nicht berücksichtigt worden, sodass sich sowohl das Ulnare als auch das Pisiforme in der herkömmlichen Deutungsweise doch als eben diese Elemente zu erkennen gäben. Die ontogenetische Untersuchung hat nun endgültig bewiesen, dass diese Auffassung wirklich zu Recht besteht. Die 1922 von mir nachgewiesenen Unstimmigkeiten finden aber ihre einfache Lösung darin, dass das bisher als Radiale bezeichnete Element zur Hauptsache ein Intermedium + Centrale proximale darstellt. 
Diese neu aufgedeckten Beziehungen stehen nun noch viel besser im Einklang mit dem von mir 1922 wahrscheinlich gemachten Aufbau des primitiven Reptilien-Basipodiums (Protorosaurus, Pelycosauria) als die damals gegebene Identifizierung, und ergeben den Beweis, dass sich in der Tat während der Ontogenese in der Crocodilier-Extremität alle Merkmale der primitiven ArchosaurierGliedmasse wiederspiegeln. Dadurch vermag diese auch, viel besser sogar, als es durch meine erstmals gegebene Deutung geschehen konnte, den Anschluss an die embryonalen Verhältnisse im Vogelflügel $\mathrm{zu}$ vermitteln. Ein Vergleich mit der embryonalen Vogelflügelanlage eines $81 / 2$ tägigen Embryos von Anser anser zeigt die grosse Aehnlichkeit, welche zwischen der ontogenetischen Entwicklung des Carpus der Vögel und jener der Krokodile besteht (vergl. Fig 7 mit Fig. 5). In beiden treten die gleichen Elemente in einer beinahe gleichen Anordnung auf. Der einzige Unterschied besteht darin, dass sich im Vogelflügel das Centrale radiale distale mit dem Centrale radiale proximale (und dem Rudiment des Radiale) zum einzigen bleibenden, radialen Handwurzelknochen der Vogelhand verbindet, während am Aufbau des entsprechenden Elementes im Carpus der Crocodilier nur das Centrale radiale proximale, das Intermedium und das Rudiment des Radiale beteiligt sind. In beiden Fällen entspricht dieses Element jedoch nicht dem Radiale der Autoren, wie es denn allgemein sehr fraglich ist, ob im Carpus und Tarsus der Sauropsida ein wirkliches Radiale sive Tibiale anders als in rudimentären Resten noch vorhanden ist. Auch mit Bezug auf die Deutung des einzigen ulnaren, zweiten Carpalknochens des Vogelflügels, des sog. Pisiforme auctorum, gibt die embryonale Entwicklung der Krokodilhand endgültigen Bescheid. Ich schrieb 1922, dass der ulnare Handwurzelknochen der Vögel zum grössten Teil eine Neubildung sei, als deren Grundlage das Metacarpale V gedient habe. Diese Beobachtung war durchaus richtig; sie ist seitdem auch von G. S. Sснеsтакоwa, 1927, für den embryonalen Flügel von Plotus levaillanti bestätigt worden. Die embryonale Krokodilhand zeigt aber (und noch viel deutlicher zeigt es der embryonale Krokodilfuss, vergl. Fig. 6), dass die proximalen Elemente des fünften Fingerstrahles (Carpale 5 und Metacarpale V) in direkten Kontakt mit der Anlage eines typischen Pisiforme treten. Im ausgebildeten Zustand liegt letzteres dagegen bei den Crocodiliern weit entfernt von der Basis des Metacarpale V 
(vergl. Fig. 3 und 4). Das Pisiforme tritt auch bei ihnen als scheinbare Neubildung später auf als die übrigen proximalen Carpalelemente. Somit liegen im Carpus der Krokodile recht ähnliche, aber offenbar ursprünglichere Verhältnisse wie im Vogelflügel vor, d.h. schon in ihm bildet sich das Pisiforme in enger Verbindung mit dem fünften Fingerstrahl. Aus diesen Zusammenhängen heraus ist aber endschliesslich zu ersehen, dass das ulnare Carpalelement des Vogelflügels doch einem echten Pisiforme entspricht (vergl.
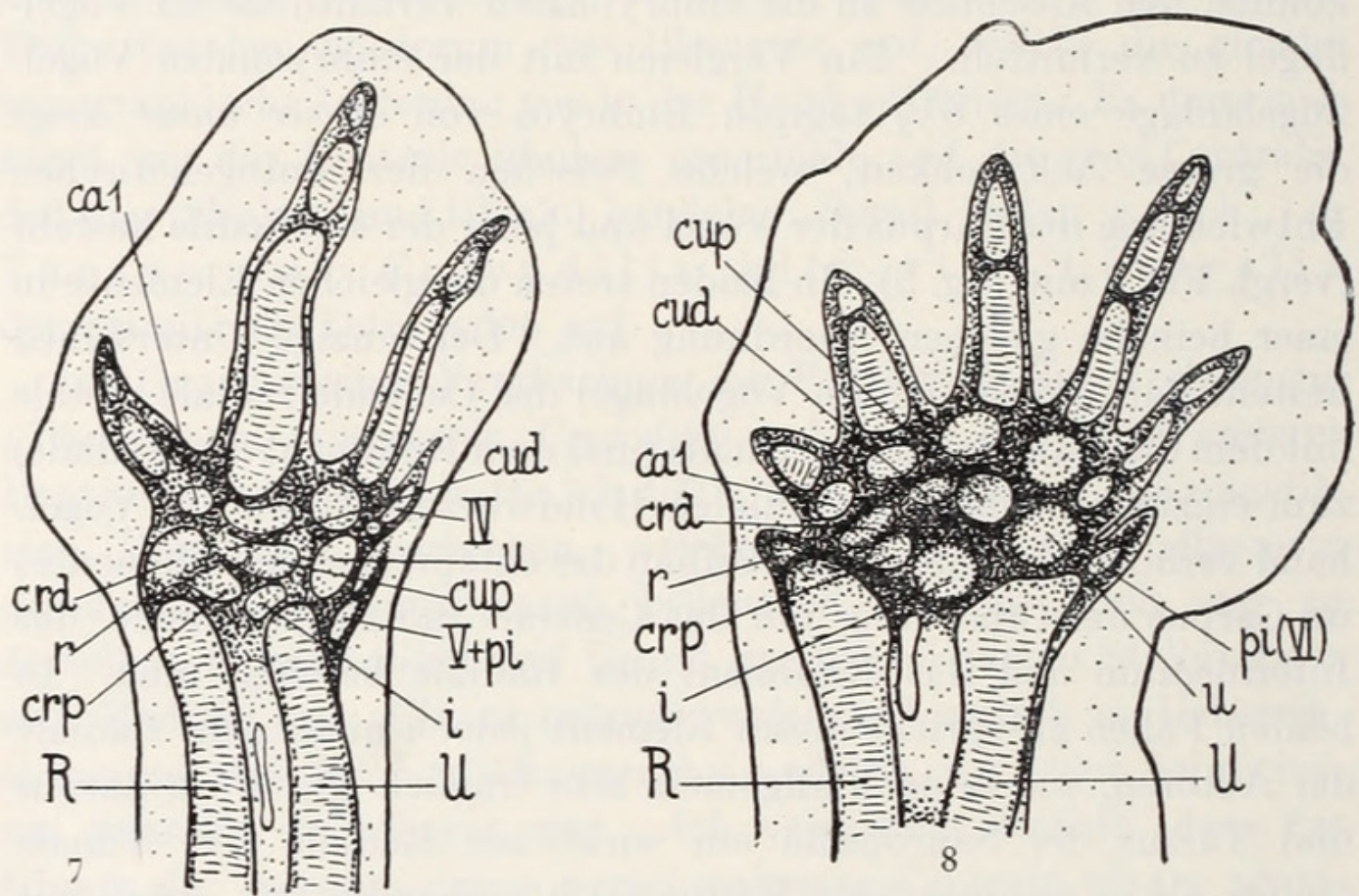

FIG. 7. - Handanlage eines 81/2 tägigen Embryos von Anser anser. Original. FIG. 8. - Handanlage eines Embryos von Chrysemys marginata. Schildlänge $6,2 \mathrm{~mm}$. Original.

Sieglbauer), in welches jedoch die rudimentäre Anlage eines fünften Fingerstrahles eingegangen ist.

Durch das Auftreten von vier Centralia während der Ontogenese der Archosaurier-Extremität werden derart altertümliche Zustände aufgedeckt, dass eine Anknüpfung an die ursprünglichste bisher bekannt gewordene Tetrapoden-Gliedmasse ermöglicht wird. Unter den lebenden Reptilien sind es hauptsächlich die Schildkröten, welche, wie schon lange bekannt ist, einen primitiven Aufbau des Extremitäten-Skeletts aufweisen. Es ist in der Tat ohne weiteres möglich, in der embryonalen Hand-oder Fussanlage einer Schildkröte ebenfalls die vier Centralia nachzuweisen, neben allen übrigen, 
schon mehrmals erwähnten Elementen (vergl. Fig. 8). Die Aehnlichkeit z.B. mit der embryonalen Fussanlage des Caiman (Fig. 6) ist eine ausserordentlich grosse. Im embryonalen Schildkröten-Basipodium ist aber ein wesentliches Merkmal in der gegenseitigen Lagerung der vier Centralia deutlicher sichtbar, das sich bei den höheren Tetrapoden immer mehr und mehr verwischt, nämlich die Anordnung in zwei nebeneinander liegende, parallele Reihen, die aus je zwei hintereinander gelegenen Elementen bestehen. Die eine Reihe geht über das Intermedium von der Ulna aus und setzt sich über das Carpale sive Tarsale 1 in den ersten Fingerstrahl fort, die zweite geht vom Ulnare aus und leitet über das Carpale sive Tarsale 2 zum zweiten Finger über. Auf diese ganz typische Anordnung habe ich schon mehrmals, 1921 und $1922 a$, hingewiesen. Ebenso ist von mir wahrscheinlich gemacht worden, dass auch den heutelebenden, niedersten Tetrapoden, den Urodelen, ursprünglich vier Centralia zukamen. Bekanntlich fand Wiedersheim, 1876, erstmals bei einigen primitiven Urodelen (Ranodon, Salamandrella, Isodactylium) zwei Centralia, welche nicht neben, sondern hintereinander lagen. Sнiткov, 1899, bestätigte auch für die larvale Entwicklung einer dieser Formen (Isodartylium) diese Angabe. Später fand aber Schmalhausen, 1910, ebenfalls bei einer dieser Arten (Salamandrella), dass embryonal sogar die Anlage von vier Centralia nachgewiesen werden kann, je zwei neben- und hintereinander. Drei und selbst vier Centralia sind seitdem öfters bei den primitivsten Urodelen (Cryptobranchus, Ambystoma, etc.) als individuelle oder regelmässige Variation beschrieben worden, wie denn speziell bei den Ambystomatiden während der Ontogenese in der Regel die Anlage von vier Centralia nachgewiesen werden kann (vergl. Fig. 9). Hierzu kommen nun noch die Befunde an den ältesten, überhaupt bekannten Tetrapoden, den Stegocephalen. Auch bei diesen ist ersichtlich, dass eine grössere Anzahl von Centralia, meistens drei, wahrscheinlich aber auch vier, im Basipodium vorhanden gewesen sind (bei Archegosaurus, Eryops, Trematops; vergl. Fig. 10). Was im Aufbau des Gliedmassenskelettes dieser Stegocephalen am meisten in die Augen springt, das ist die geradezu verblüffende Aehnlichkeit, wenn nicht Gleichheit, mit jenem der recenten Urodelen, hauptsächlich mit ihren ursprünglichsten Typen (Cryptobranchus, Ambystoma, Ranodon, etc.). Insbesondere sind die Centralia 
ebenfalls in zwei Reihen von je zwei hintereinander gelegenen Elementen angeordnet. Nichts anderes aber fanden wir ebenfalls im Basipodium der Chelonier (Fig. 8) und konnten wir noch in der Extremität der Krokodile und der Vögel nachweisen. So gelingt es in der Tat, selbst diese hochspezialisierten und einseitig einer bestimmten Funktion angepassten Gliedmassen höherer Tetrapoden ohne Zwang auf die ursprünglichste Vierfüsser-Extremität zurückzuführen.
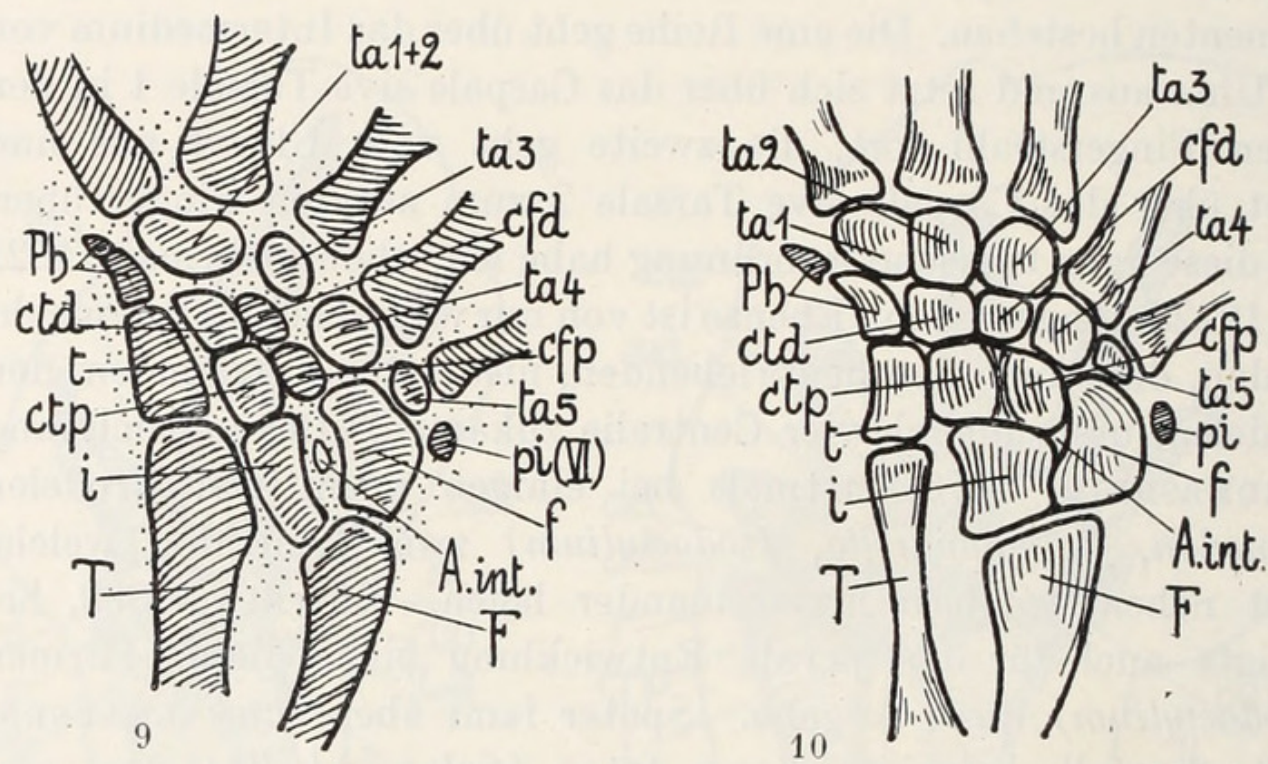

FIG. 9. - Fussanlage einer Larve von Cryptobranchus japonicus. Original. Alle Elemente noch rein knorpelig.

FIg. 10. - Primitivster Basipodium-Typus. In Anlehnung an den Tarsus von Trematops milleri Williston, 1909. Alle Elemente verknöchert; supponierte knorpelige Elemente schraffiert.

(Durch einen jener so häufigen Fälle der Duplizität hat ausser meinen neuen Untersuchungen auch N. Holmgren, erstmals seit der Publikation von 1893 von Kuekenthal, die Krokodil-Extremität einer neuerlichen embryologischen Prüfung in einer 1933 erschienenen Arbeit über den Ursprung der Tetrapoden-Gliedmasse unterzogen. Ich habe erst nach Abschluss meiner Untersuchungen Kenntnis von seinen Resultaten erhalten, welche, was diē Anzahl und die Homologisierung der einzelnen Elemente des KrokodilBasipodiums anbetrifft, im Wesentlichen mit meinen Befunden übereinstimmen. In der Deutung seiner Ergebnisse weicht aber Holmgren bedeutend von den hier vertretenen Anschauungen ab. Es soll andernorts näher darauf eingetreten werden.) 


\section{ERKLÄRUNG DER IN DEN TEXTFIGUREN GEBRAUCHTEN ABKÜRZUNGEN}

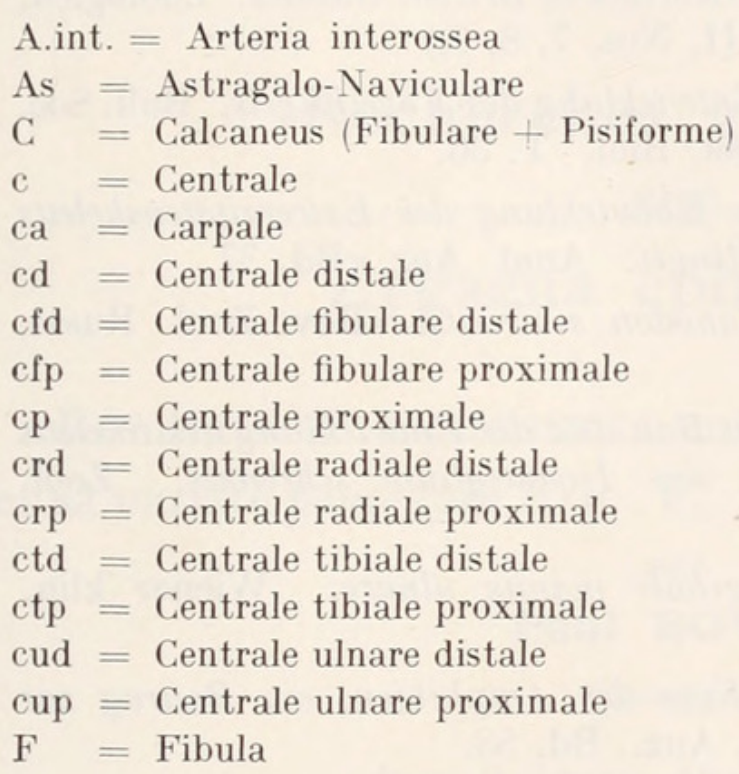

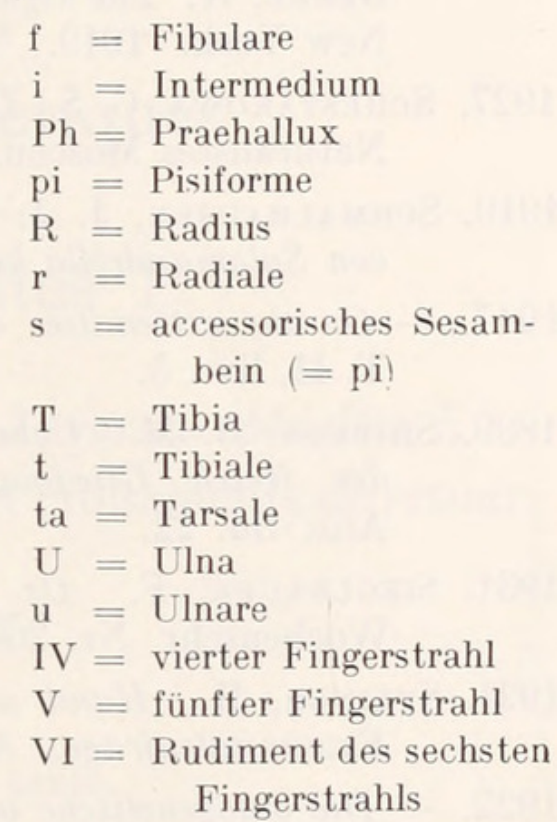

$\mathrm{f}=$ Fibulare

$\mathrm{i}=$ Intermedium

$\mathrm{Ph}=$ Praehallux

pi $=$ Pisiforme

$\mathrm{R}=$ Radius

$\mathrm{r}=$ Radiale

$\mathrm{s}=$ accessorisches Sesambein $(=\mathrm{pi})$

$\mathrm{T}=$ Tibia

ta $=$ Tarsale

$\mathrm{U}=$ Ulna

$\mathrm{u}=$ Ulnare

$\mathrm{IV}=$ vierter Fingerstrahl

$\mathrm{V}=$ fünfter Fingerstrahl Fingerstrahls

\section{ERWÄHNTE LITERATUR}

1876. Bonn, G. Zum Carpus und Tarsus der Saurier. Morph. Jahrb. Bd. 2.

1824. Cuvier, G. Recherches sur les ossemens fossiles. T. 5, II e partie. 1864. Gegenbaur, C. Untersuchungen zur vergl. Anatomie der Wirbeltiere. 1. Heft. Carpus und Tarsus.

1890. Hofmann, C. K. Reptilien II. Eidechsen und Wasserechsen. In: Bronn's Klassen und Ordnungen des Tierreichs. Bd. 6, Abt. III.

1933. Holmgren, N. On the origin of the tetrapod limb. Acta Zool. Bd. 14.

1914. v. Huene, F. Beiträge zur Geschichte der Archosaurier. Geol. Paläont. Abh. N. F. Bd. 13.

1920. - Stammesgeschichtliche Ergebnisse einiger Untersuchungen an Trias-Reptilien. Zeitschr. ind. Abst.-Vererbl. Bd. 24.

1927. - Die Beziehungen zwischen den paläozoischen und den mesozoischen Reptilien. Paläont. Zeitschr. Bd. 9. 
1893. Kuekenthal, W. Zur Entwicklung des Handskelettes des Krokodils. Morph. Jahrb. Bd. 19.

1824. Meckel, J. F. System der vergleichenden Anatomie. Bd. 2, Abt. I.

1915. Reese, A. M. The alligator and his allies. New York. (Siehe auch BeEne, W. The higher vertebrates of British Guiana. Zoologica. New York. 1919. Vol. II, Nos. 7, 8, 9.)

1927. Schestakowa, G. S. Die Entwicklung des Vogelflügels. Bull. Soc. Naturalistes Moscou. Sect. Biol. T. 36.

1910. Schmalhausen, J. J. Die Entwicklung des Extremitätenskeletts von Salamandrella keyserlingii. Anat. Anz. Bd. 37.

1917. - On the extremities of Ranodon sibiricus. Rev. Zool. Russe. T. II, livr. 5.

1899. Sнiтkov. B. M. Ueber den Bau und die Entwicklung des Skeletts der freien Gliedmassen des Isodactylium schrenkii. Zool. Anz. Bd. 22.

1931. Sieglbauer, F. Os marginale manus ulnare. Wiener klin. Wochenschr. Nr. 26.

1921. Steiner, H. Hand und Fuss der Amphibien, ein Beitrag zur Extremitätenfrage. Anat. Anz. Bd. 53.

1922. - Die ontogenetische und phylogenetische Entwicklung des Vogelflügelskelettes. Acta Zool. Bd. 3.

1922a. - Der ursprüngliche Aufbau des Extremitätenskeletts der Tetrapoden. Verhandl. Schweiz. Naturf. Ges. Bern. Teil II.

1876. Wiedersheim, R. Die ältesten Formen des Carpus und Tarsus der heutigen Amphibien. Morph. Jahrb. Bd. 2 (siehe auch Bd. 3 und 6$)$.

1909. Williston, S. W. New or little known Permian Vertebrates. Trematops. Journ. Geol. Chicago. Vol. 17. 


\section{$2 \mathrm{BHL}$ Biodiversity Heritage Library}

Steiner, H. 1934. "Ueber die embryonale Hand- und Fuss-Skelett-Anlage bei den Crocodiliern, sowie über ihre Beziehungen zur Vogel-Flügelanlage und zur ursprünglichen Tetrapoden-Extremität." Revue suisse de zoologie 41, 383-396. https://doi.org/10.5962/bhl.part.146012.

View This Item Online: https://www.biodiversitylibrary.org/item/177005

DOI: https://doi.org/10.5962/bhl.part.146012

Permalink: https://www.biodiversitylibrary.org/partpdf/146012

\section{Holding Institution}

American Museum of Natural History Library

\section{Sponsored by}

BHL-SIL-FEDLINK

\section{Copyright \& Reuse}

Copyright Status: Public domain. The BHL considers that this work is no longer under copyright protection.

Rights Holder: Muséum d'histoire naturelle - Ville de Genève

This document was created from content at the Biodiversity Heritage Library, the world's largest open access digital library for biodiversity literature and archives. Visit BHL at https://www.biodiversitylibrary.org. 